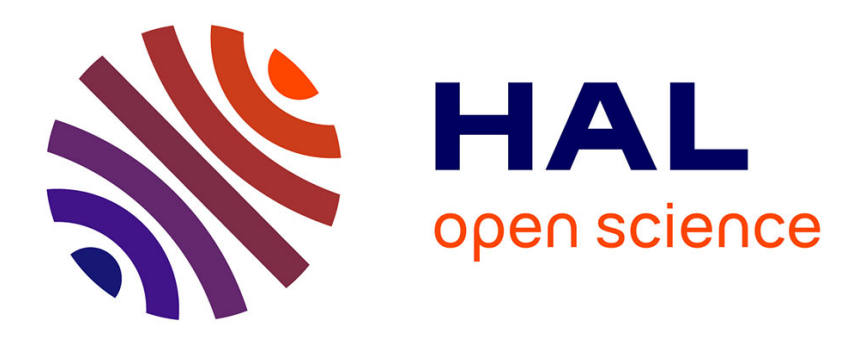

\title{
Vibration Reduction in Piecewise Bi-Coupled Periodic Structures
}

\author{
Francesco Romeo, Angelo Luongo
}

\section{To cite this version:}

Francesco Romeo, Angelo Luongo. Vibration Reduction in Piecewise Bi-Coupled Periodic Structures. Journal of Sound and Vibration, 2003, 268 (3), pp.601-615. hal-00795116

\section{HAL Id: hal-00795116 https://hal.science/hal-00795116}

Submitted on 27 Feb 2013

HAL is a multi-disciplinary open access archive for the deposit and dissemination of scientific research documents, whether they are published or not. The documents may come from teaching and research institutions in France or abroad, or from public or private research centers.
L'archive ouverte pluridisciplinaire HAL, est destinée au dépôt et à la diffusion de documents scientifiques de niveau recherche, publiés ou non, émanant des établissements d'enseignement et de recherche français ou étrangers, des laboratoires publics ou privés. 


\title{
Vibration reduction in piecewise bi-coupled periodic structures
}

\author{
F. Romeo ${ }^{\mathrm{a}}$, A. Luongo ${ }^{\mathrm{b}, *}$
}

\author{
a Dipartimento di Ingegneria Strutturale e Geotecnica, Università di Roma "La Sapienza", Via Gramsci 53, \\ 197 Roma, Italy \\ ${ }^{\mathrm{b}}$ Dipartimento di Ingegneria delle Strutture, delle Acque e del Terreno (DISAT), Università di L'Aquila, \\ 67040 Monteluco di Roio, L'Aquila, Italy
}

\begin{abstract}
The problem of minimizing transmitted vibrations through finitely long periodic structures is addressed. Bi-coupled periodic element properties and arrangement are tailored to localize the response around the excitation source within any assigned frequency range. Bi-dimensional analytical maps of the single unit free-wave propagation domains (stop, pass and complex domains) provide the optimal choice of the cell properties and ordering. Moreover, the amount of vibration suppression along the periodic structure is also controlled as it can be described through iso-attenuation curves representing the contour plot of the real part of the propagation constants. Applications to both undamped and damped beams resting on elastic supports are illustrated. The response of the periodic structures to harmonic excitations is expressed through the wave vector method taking into account the effects of wave reflection due to changes in the cell properties along the structure and boundary conditions. Such computational schemes enables one to overcome numerical difficulties arising in the transfer matrix formulation for structures with a large number of periodic units.
\end{abstract}

\section{Introduction}

Several authors have investigated the possibility of reducing transmitted vibrations in periodic structures. In Ref. [1], the optimal design of beams on multiple supports to minimize vibration transmission and stress levels has been pursued. An optimization procedure has been employed to select suitable slight deviations from periodicity, considering as design parameters the individual bay lengths and damping values. On the other hand, in Refs. [2,3], vibrations have been reduced

\footnotetext{
*Corresponding author. Tel.: + 390862 434511; fax: + 390862434548.

E mail addresses: francesco.romeo@uniroma1.it (F. Romeo), luongo@ing.univaq.it (A. Luongo).
} 
by introducing intentional spatial periodicity in the otherwise non-periodic structure and resorting to their propagation properties. In the first work, the authors propose to design periodically supported piping system. In the latter, aiming at reducing gear mesh vibration, a periodic shaft has been used to design stop bands in the frequency spectra that correspond to particular harmonics of the gear mesh contact dynamics; both numerical and experimental results have shown significant vibration reduction. Recently, in Ref. [4], pass and stop bands of mono-coupled periodic systems made up of masses connected by mechanical springs have been shown to be controllable by adding active piezoelectric springs; moreover, vibration localization has been obtained by randomly disordering the control gains of each active spring. All of the above analyses have merely relied upon ad hoc numerical investigations on the propagation properties of the single periodic units in order to optimize the design. Moreover, the influence of the physical parameters on the amount of vibration reduction has not been fully investigated. More recently, in Ref. [5], bi-coupled periodic structures have been analyzed on the basis of the transfer matrix characteristic equation and analytical maps of the single unit free-wave propagation domains (stop, pass and complex domains) have been derived. From such maps, the role played by the physical (control) parameters in the wave propagation properties can be readily gathered and thereby exploited. Based on these results, aiming at reducing the transmitted vibrations, a design of optimal piecewise periodic structures is proposed in this work. In particular, the periodic cell properties and arrangement are tailored to localize the response around the excitation source within any assigned frequency range. The amount of vibration suppression along the periodic structure is also controlled as it can be described through iso-attenuation curves representing the contour plot of the real part of the propagation constants. Numerical applications carried-out by means of the wave vector approach are illustrated for beams resting on elastic supports. The adopted computational scheme has been devised [ 6 r 8 ] to overcome the numerical difficulties arising in the transfer matrix formulation $[9,10]$ when the number of periodic units increases; in particular, the frequency-dependent global transfer matrix, connecting the state variables at the ends, becomes ill-conditioned since the ratio between its maximum and minimum eigenvalue increases as well. This problem can be physically interpreted by considering that the transfer matrix implies rightwards transmission. Therefore, backward decaying waves appear numerically as growing waves in the opposite direction, thereby amplifying unavoidable numerical errors. Periodic structures and structures whose periodicity patterns change from one section of the structure to another (piecewise periodic structures) are analyzed by transforming traditional transfer matrices for state vectors to transfer matrices for wave vectors enabling the computations to always proceed in the direction of wave motion.

\section{Analytical model and design strategy}

In Ref. [5], bi-coupled periodic structures have been analyzed on the basis of the invariants $I_{j}(j=1,2)$ of the transfer matrix $\mathbf{T}$ and analytical results on the single unit free-wave (characteristic waves) propagation properties have been derived. According to that paper, an exhaustive geometrical representation of such propagation properties is achieved by identifying in the two-dimensional space $\left\{I_{j}\right\}$ the domains in which the four eigenvalues $\lambda$ of $\mathbf{T}$ are of the same type. The regions where both the pairs of $\lambda$ lay on the unit circle are referred to as pass-pass (PP); 
the regions where only one pair of $\lambda$ lays on the unit circle while the other pair is real are referred to as pass-stop (PS); the regions where only real pairs of eigenvalues occur are the stop-stop (SS) domains. Moreover, complex regions $(\mathrm{C})$ exist where the eigenvalues are complex conjugate.

In order to analyze a specific bi-coupled structure, such universal description in the invariant space is transformed into a physical space by expressing the invariants $I_{j}$ as functions of two control parameters. In this work the attention is focused on bi-coupled periodic structures whose repetitive elements, as sketched in Fig. 1, are given by Euler beams of length $l$, flexural stiffness $E I$, resting on elastic supports with translational stiffness $k_{t} / 2$, with distributed mass $m$. For such periodic elements, the state vector at the coupling point $k$ is given by $\mathbf{z}_{k}=\left(v_{k}, \varphi_{k}, V_{k}, M_{k}\right)^{\mathrm{T}}$, where $v, \varphi$ and $V, M$ represent the generalized displacement and force components, respectively. The selected control parameters governing the propagation properties of a cell are the nondimensional frequency and spring stiffness defined as

$$
\beta=\sqrt[4]{m \omega^{2} l^{4} /(E I), \quad \kappa=k_{t} l^{3} / E I .}
$$

The dependence of the free-wave propagation characteristics on the spring translational stiffness and frequency is described in Fig. 2a where the propagation regions map is shown. In the pass regions waves propagate harmonically without attenuation, whereas in the stop regions waves decay; harmonic propagation with attenuation occurs in the complex regions. The branches $r_{i}, s_{i}$ and $p_{i}$ bounding the different type of regions have been analytically derived in Ref. [5]; the curves $r_{i}$ and $s_{i}$ are given by

$$
r_{i}, s_{i}:=\left\{(\beta, \kappa) \mid \begin{array}{ll}
1 \pm \cos \beta=0 & \text { for } i \text { odd } \\
\kappa=\frac{\mp 4 \beta^{3}(1 \pm \cosh \beta)}{\frac{\sin \beta}{(1 \pm \cos \beta)}(1 \pm \cosh \beta) \sinh \beta} & \text { for } i \text { even }
\end{array}\right\}
$$

while the curves $p_{i}$ are made up of two branches, $p_{i u}$ (upper) and $p_{i l}$ (lower), defined in the intervals $\beta \in[n \pi,(n+1) \pi]$ ( $n$ even), having equations

$$
p_{i u, l}:=\left\{(\beta, \kappa) \mid \kappa=\frac{4 \beta^{3}(\cosh \beta \quad \cos \beta)}{(\sqrt{ } \sin \beta \pm \sqrt{ } \sinh \beta)^{2}}\right\} .
$$

Aiming at reducing the transmitted vibrations induced by an excitation with known frequency bandwidth, the previous findings can be used to delineate a design strategy. This consists in realizing a piecewise periodic structure made up of the minimum number of dissimilar sections such that the union of the stop and complex propagation bands of each element covers the whole frequency range of interest, namely the excitation bandwidth. Such sequence of periodic sections will be referred to as optimal piecewise periodic structure. Starting from $\beta=0$, a sequence of optimal values $\kappa^{*}$ is determined as illustrated by the stepwise dashed line in Fig. 2a. This is obtained by selecting the values $\kappa_{i}^{*}$ relevant to the intersections between curves $r_{i}$ and $s_{i}$ (Fig. 2b)

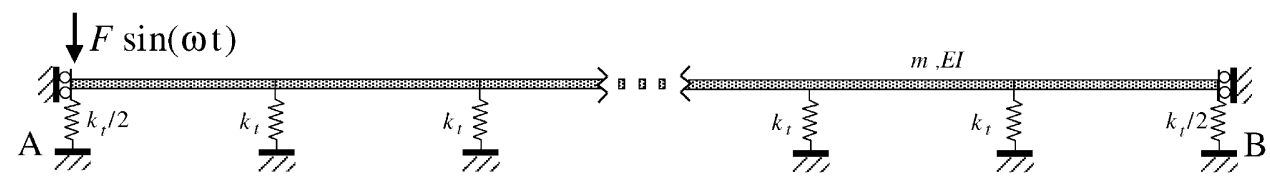

Fig. 1. Uniform Euler beam on evenly spaced springs. 


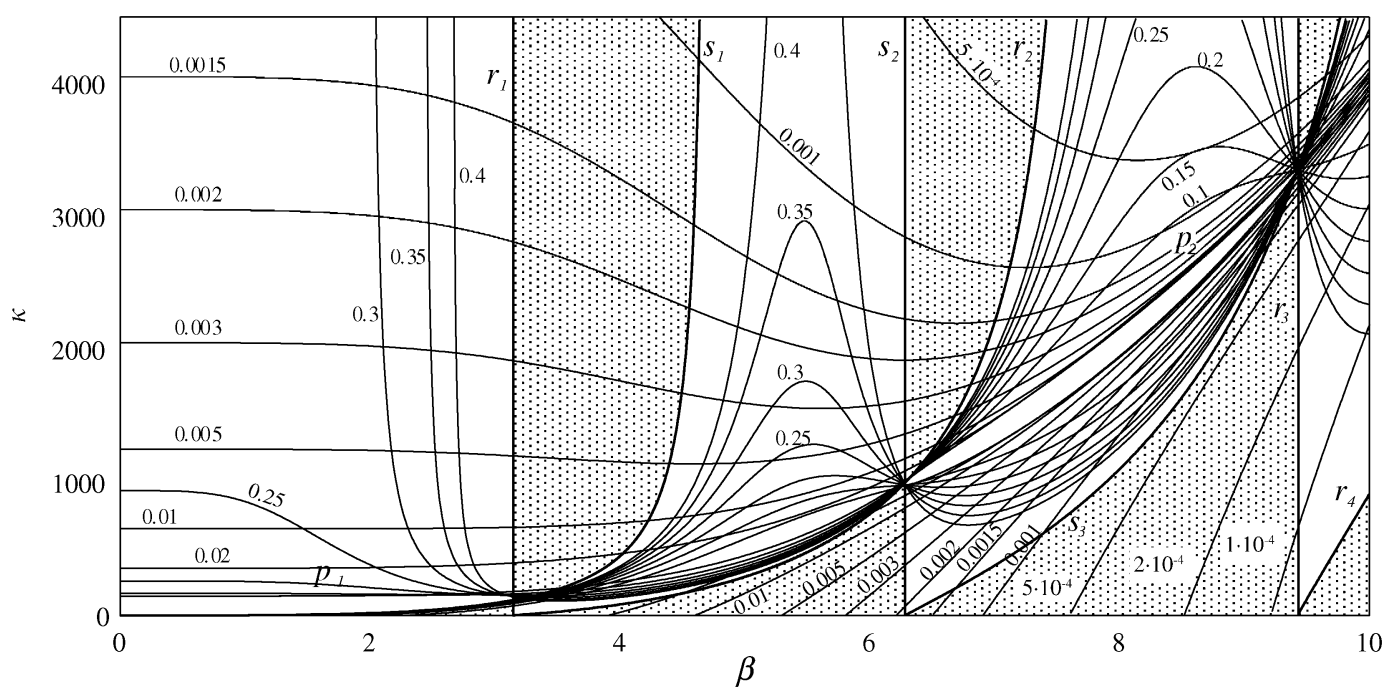

Fig. 3. Iso attenuation curves on the $\beta \kappa$ plane.

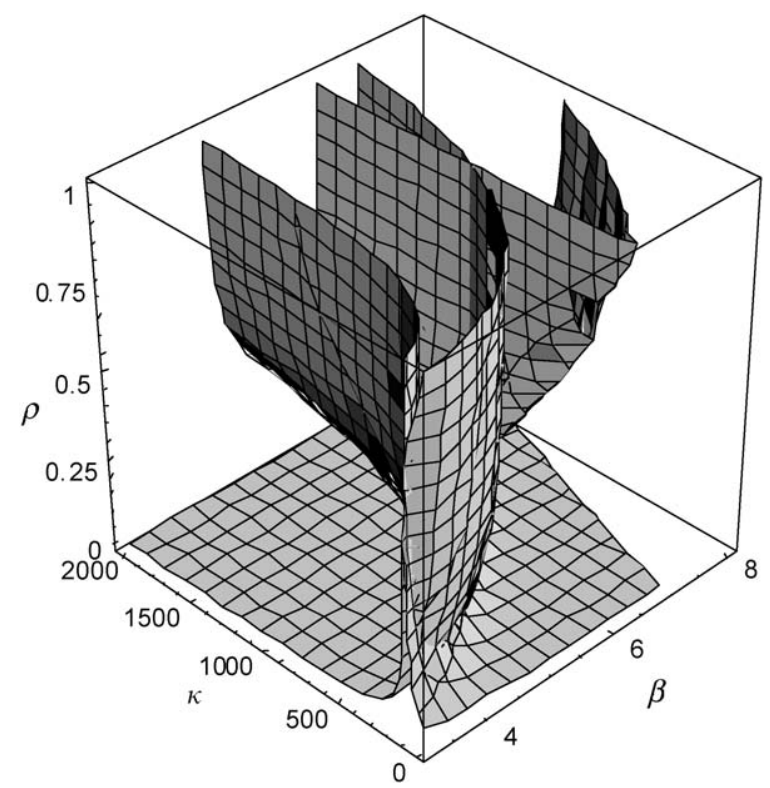

Fig. 4. Modulus $\rho$ of the eigenvalues of T: surface $\rho=\rho(\beta, \kappa)$.

The outlined strategy prevents vibration transmission at all frequencies within the design frequency range, except for the natural frequencies, where resonance takes place. The natural frequencies of the overall piecewise periodic structure lay within the union of either pass pass and/or pass stop bands of each section. Therefore, piecewise periodic structures are characterized by lower modal density than uniformly periodic ones with the same number of elements, this 
entailing a further beneficial effect on the response. In the ideal undamped structure the reduction of vibration propagation along the structure implies narrowing of the resonances while the amplification remains ideally infinite. On the other hand, in real structures a small amount of damping limits the resonance amplifications; therefore, not only the response at generic frequencies but also the resonance peaks are lowered.

\section{Vibration analysis using wave vectors}

In the vibration analysis of periodic and piecewise periodic structures, summarized in this section, structural members are treated as waveguides which transmit a disturbance from one location to another as wave motion (see Fig. 5). According to this approach, the equations governing the problem are derived by meeting transmission, continuity and boundary conditions. The starting point of the computational scheme is the transformation of state vectors to wave vectors. Next, continuity conditions at the interface of dissimilar cells as well as boundary conditions are introduced in terms of wave coordinates.

For piecewise periodic structures the generic section $\alpha$ is composed of $N_{\alpha}$ elements connecting nodes $i$ and $j$; according to the transfer matrix approach the state vector at the coupling point $j$ is related to the state vector at the coupling point $i$ by

$$
\left(\begin{array}{c}
\mathbf{x}_{j} \\
\mathbf{f}_{j}
\end{array}\right)=\mathbf{T}_{\alpha}^{N_{\alpha}}\left(\begin{array}{c}
\mathbf{x}_{i} \\
\mathbf{f}_{i}
\end{array}\right),
$$

where, if the elements are coupled through $n$ degrees of freedom, $\mathbf{T}_{\alpha}$ is the $(2 n \times 2 n)$ frequencydependent transfer matrix of the single element of section $\alpha$. A peculiar property of the transfer matrix is that its eigenvalues are reciprocal pairs $(\lambda, 1 / \lambda)$. The $2 n$ state vector can be transformed to wave vector through the matrix $\mathbf{U}_{\alpha}$ whose columns are eigenvectors of $\mathbf{T}_{\alpha}$ as follows:

$$
\left(\begin{array}{c}
\mathbf{x}_{i} \\
\mathbf{f}_{i}
\end{array}\right)=\mathbf{U}_{\alpha}\left(\begin{array}{c}
\mathbf{r}_{i j} \\
\mathbf{l}_{i j}
\end{array}\right) .
$$

As depicted in Fig. 5, the terms $\mathbf{r}$ and $\mathbf{I}$ hints at right- and left-going waves, respectively. They represent the amplitudes of the eigenvectors associated to reciprocal pairs with $|\lambda|<1$ and $|\lambda|>1$, respectively; if $|\lambda|=1$, the two reciprocal eigenvalues are complex conjugate and the associated amplitudes split in $\mathbf{r}$ and $\mathbf{l}$. Substituting Eq. (6) in Eq. (5) gives

$$
\begin{aligned}
& \left(\begin{array}{c}
\mathbf{r}_{j i} \\
\mathbf{l}_{j i}
\end{array}\right)=\mathbf{U}_{\alpha}{ }^{1} \mathbf{T}_{\alpha}^{N_{\alpha}} \mathbf{U}_{\alpha}\left(\begin{array}{c}
\mathbf{r}_{i j} \\
\mathbf{l}_{i j}
\end{array}\right)=\left[\begin{array}{cc}
\boldsymbol{\Lambda}_{\alpha}^{N_{\alpha}} & 0 \\
0 & \boldsymbol{\Lambda}_{\alpha} N_{\alpha}
\end{array}\right]\left(\begin{array}{l}
\mathbf{r}_{i j} \\
\mathbf{l}_{i j}
\end{array}\right),
\end{aligned}
$$

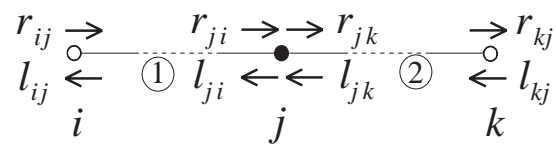

Fig. 5. Two way junction of dissimilar elements and corresponding wave co ordinates: $\bullet$, external constraint; $\bullet$, internal constraint. 
where $\boldsymbol{\Lambda}_{\alpha}$ is a diagonal matrix collecting the eigenvalues with $|\lambda| \leqslant 1$. Rearranging Eq. (7) to follow the wave propagation direction,

$$
\left(\begin{array}{c}
\mathbf{r}_{j i} \\
\mathbf{l}_{i j}
\end{array}\right)=\left[\begin{array}{cc}
\boldsymbol{\Lambda}_{\alpha}^{N_{\alpha}} & 0 \\
0 & \boldsymbol{\Lambda}_{\alpha}^{N_{\alpha}}
\end{array}\right]\left(\begin{array}{c}
\mathbf{r}_{i j} \\
\mathbf{l}_{j i}
\end{array}\right) .
$$

The state vector at the interface of dissimilar cells, such as the coupling point $j$ between nodes $i$ and $k$ (see Fig. 5), must be continuous in the sense that displacements must be equal and forces must be in equilibrium, as long as no excitation is applied at the interface. Such continuity requirement is expressed relating the outgoing waves at the interfaces of cells to the incoming ones as follows:

$$
\left(\begin{array}{c}
\mathbf{l}_{j i} \\
\mathbf{r}_{j k}
\end{array}\right)=\left[\begin{array}{ll}
\mathbf{S}_{l r} & \mathbf{S}_{l l} \\
\mathbf{S}_{r r} & \mathbf{S}_{r l}
\end{array}\right]\left(\begin{array}{c}
\mathbf{r}_{j i} \\
\mathbf{l}_{j k}
\end{array}\right),
$$

where the wave scattering matrix $\mathbf{S}$ at the interface $j$ has been partitioned in reflection $\left(\mathbf{S}_{l r}, \mathbf{S}_{r l}\right)$ and transmission $\left(\mathbf{S}_{l l}, \mathbf{S}_{r r}\right)$ submatrices.

Now refer to the two-way junction shown in Fig. 5 excited by a harmonic forces $\mathbf{f}_{i}$ applied at node $i$; let $N_{1}=N$ and $N_{2}=M$ be the number of elements of Sections 1 and 2, respectively. The boundary conditions at the ends of the periodic chains allows one to express the outgoing waves in terms of the incoming ones by means of reflection matrices $\mathbf{R}$ as follows:

$$
\mathbf{l}_{i j}=\mathbf{R}_{i} \mathbf{r}_{i j}+\mathbf{D f} \mathbf{f}_{i}, \quad \mathbf{r}_{k j}=\mathbf{R}_{k} \mathbf{l}_{k j} .
$$

Details on the derivation of the matrices $\mathbf{R}(n \times n), \mathbf{S}(2 n \times 2 n)$ and $\mathbf{D}(n \times n)$ can be found in the appendix. By combining Eqs. (8) (10) any arrangement of elements at a junction can be solved in terms of the unknown wave vectors. The core of the computational scheme consists of solving the above-mentioned sets of equations by condensing the whole unknown wave vector in only the components entering the domain. Using Eqs. (8) and (9) to eliminate the outgoing waves at the ends and all the waves at node $j$, the resolving Eq. (10) are obtained in terms of waves entering the domain

$$
\left[\begin{array}{cc}
\mathbf{R}_{i}+\boldsymbol{\Lambda}_{1}^{N} \mathbf{S}_{l r} \boldsymbol{\Lambda}_{1}^{N} & \boldsymbol{\Lambda}_{1}^{N} \mathbf{S}_{l l} \boldsymbol{\Lambda}_{2}^{M} \\
\boldsymbol{\Lambda}_{2}^{M} \mathbf{S}_{r r} \boldsymbol{\Lambda}_{1}^{N} & \mathbf{R}_{k}+\boldsymbol{\Lambda}_{2}^{M} \mathbf{S}_{r l} \boldsymbol{\Lambda}_{2}^{M}
\end{array}\right]\left(\begin{array}{c}
\mathbf{r}_{i j} \\
\mathbf{I}_{k j}
\end{array}\right)=\left(\begin{array}{c}
\mathbf{D} \mathbf{f}_{i} \\
\mathbf{0}
\end{array}\right) .
$$

The wave co-ordinates at the intermediate nodes can be derived and transformed back to obtain the response expressed by state variables; next, the response of the elements is readily evaluated as a function of the ends' displacements and rotations. The homogeneous form of problem (11) furnishes the natural frequencies and the "mode" $\left(\mathbf{r}_{i j}, \mathbf{l}_{k j}\right)^{\mathrm{T}}$.

When the groups of elements between nodes $i$ and $k$ are identical, the scattering matrix becomes simply

$$
\mathbf{S}=\left[\begin{array}{ll}
\mathbf{0} & \mathbf{I} \\
\mathbf{I} & \mathbf{0}
\end{array}\right]
$$


meaning that the continuity at the junction must be met not only by the state vector but also by the wave vector. Consequently, system (11) becomes

$$
\left[\begin{array}{cc}
\mathbf{R}_{i} & \boldsymbol{\Lambda}^{N+M} \\
\boldsymbol{\Lambda}^{M+N} & \mathbf{R}_{k}
\end{array}\right]\left(\begin{array}{l}
\mathbf{r}_{i j} \\
\mathbf{l}_{k j}
\end{array}\right)=\left(\begin{array}{c}
\mathbf{D} \mathbf{f}_{i} \\
\mathbf{0}
\end{array}\right) .
$$

\section{Numerical results}

Uniform undamped Euler beams supported by evenly spaced springs (Fig. 1) have been considered in the numerical investigations. The ends are constrained by sliding supports and a harmonic excitation is applied at the left end of the periodic beam. According to the design strategy outlined in Section 2, piecewise periodicity is introduced to reduce transmitted vibrations in a given frequency range. Willing to reduce the transmitted vibrations induced by excitations with frequency starting from $\beta=0$, the first optimal value is $\kappa_{1}^{*}$ (Fig. 2). Adopting this value of the spring translational stiffness a pass pass region $(3.092 \leqslant \beta \leqslant \pi)$ is found between a complex $(0 \leqslant \beta<3.092)$ and a stop stop $(\pi \leqslant \beta \leqslant 4.220)$ region; then, a pass stop band ending at $\beta=2 \pi$ exists. Therefore by composing the whole structure with this type of element, attenuating vibrations up to $\beta=3.092$ are assured. In order to extend the design frequency range beyond $\beta=3.092$, the optimal value $\kappa_{12, \text { max }}^{*}$ must be taken into account, thus assuring vibration reduction up to $\beta=5.908$. Further extension of the design frequency interval for vibration reduction is achieved by using three types of elements of stiffness, from left to right, $\kappa_{1}^{*}, \kappa_{12, \max }^{*}, \kappa_{2}^{*}$, thus covering the frequency range $0 \leqslant \beta \leqslant 7.886$. If the design goal is to reduce transmitted vibrations starting from $\beta=7.886$ to 0 , then the optimal sequence from left to right would be $\kappa_{2}^{*}, \kappa_{12, \text { min }}^{*}, \kappa_{1}^{*}$. In principle, any value $\kappa_{12, \min }^{*} \leqslant \kappa_{12}^{*} \leqslant \kappa_{12, \max }^{*}$ guarantees the overlay of the frequency range with either stop or complex bands. Thus both the problems of the choice of $\kappa_{12}^{*}$ and of the spatial arrangement of the sections arise. Concerning this, it is worth noticing that the frequency response belonging to the stop band of the section closest to the excitation source exhibit the fastest spatial decay rate. Therefore, aiming at minimizing the spatial extension of the response in the lowfrequency range, the sections must be ordered with increasing stiffnesses, selecting $\kappa_{12}^{*}=\kappa_{12, \max }^{*}$. Otherwise, to minimize the extension of the high-frequency response, the sections must be ordered with decreasing stiffnesses, selecting $\kappa_{12}^{*}=\kappa_{12, \text { min }}^{*}$. Indeed, such choices of $\kappa_{12}^{*}$ extend at most upwards and downwards, respectively, the frequency range inhibited by the intermediate section.

The numerical values of the optimal sequence of $\kappa^{*}$ for $0 \leqslant \beta \leqslant 11.308$ are reported in Table 1 . The frequency value in each row represents the bound of the range controlled by using elements with $\kappa^{*}$ up to the actual one.

For a six-span beam made up by equal elements $\kappa_{1}^{*}$, the displacement magnification factors $D_{A}$ and $D_{B}$ at the ends of the beam are shown in Figs. 6a and b, respectively. As expected, by considering the range $0 \leqslant \beta \leqslant 6$, the vibration transmission from node $A$ to node $B$ takes place within the pass pass and pass stop bands where two groups of six natural frequencies are also found. The amount of attenuation varies as the frequency grows. Specifically, by focusing on the intervals between the spikes of the natural frequencies in the PS band in Fig. 6b, the magnification factor $D_{B}$ shows a decaying trend. Such behavior can also be inferred by the arrangement of the 


\section{Table 1}

Optimal sequence of $\kappa(0 \leqslant \beta \leqslant 11.308)$ and associated controlled frequency range bounds; (above) increasing frequencies, (below) decreasing frequencies

\begin{tabular}{lrr}
\hline & \multicolumn{1}{l}{$\kappa^{*}$} & $\beta$ \\
\hline$\kappa_{1}^{*}$ & 113.750 & 3.092 \\
$\kappa_{12, \max }^{*}$ & 696.500 & 5.908 \\
$\kappa_{2}^{*}$ & 995.914 & 7.886 \\
$\kappa_{3}^{*}$ & 3348.137 & 11.308 \\
$\kappa_{3}^{*}$ & 3348.137 & 7.272 \\
$\kappa_{2}^{*}$ & 995.914 & 4.355 \\
$\kappa_{12, \min }^{*}$ & 136.823 & 3.265 \\
$\kappa_{1}^{*}$ & 113.750 & 0 \\
\hline
\end{tabular}
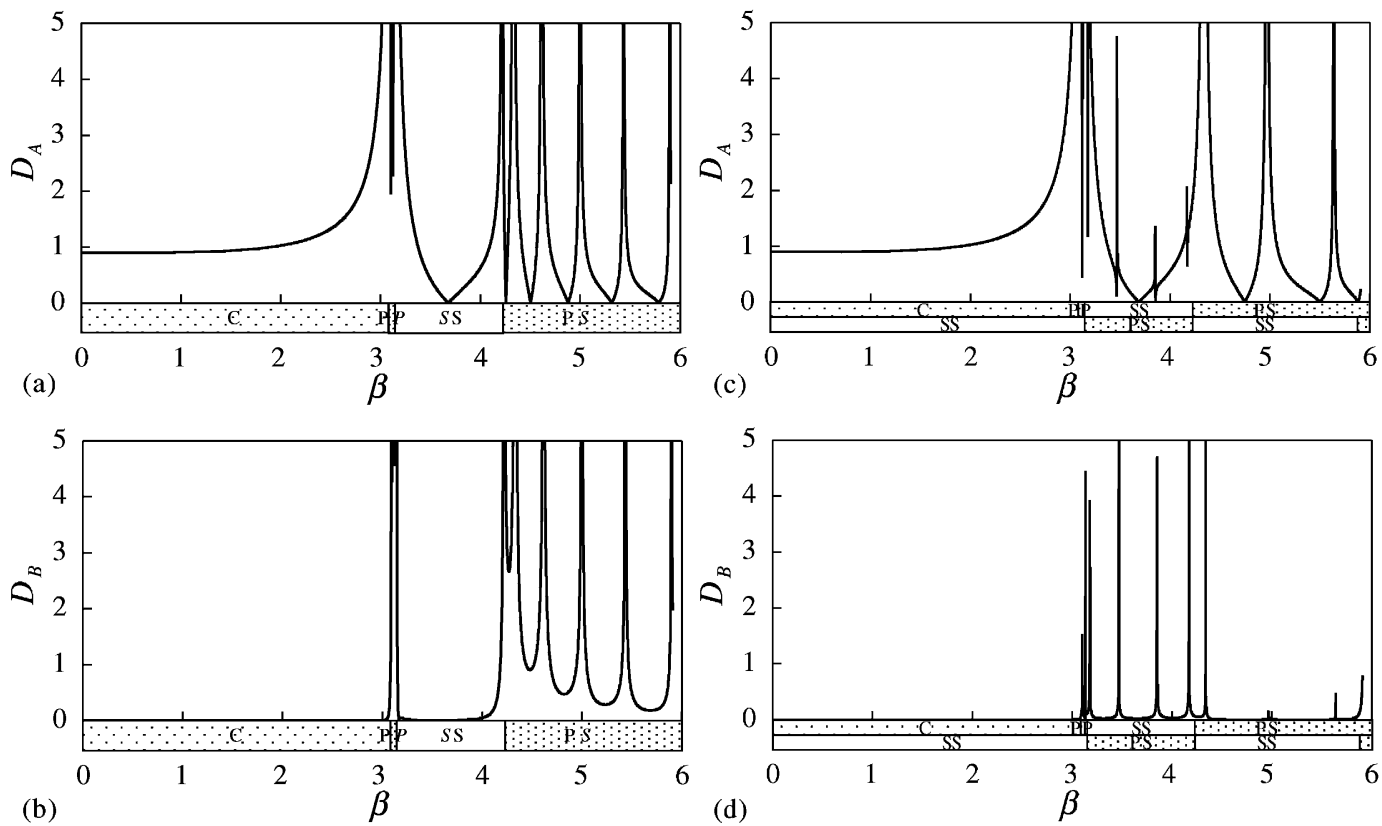

Fig. 6. Displacement dynamic magnification factors: ((a), (b)) $\kappa_{i}=\kappa_{1}^{*}$, ((c), (d)) $\kappa_{1,2,3}=\kappa_{1}^{*}, \kappa_{4,5,6}=\kappa_{12, \max }^{*}$; ((a) (c)) node $A$, ((b) (d)) node $B$.

iso-attenuation curves illustrated in Fig. 4. Figs. $6 \mathrm{c}$ and d show the magnification factors $D_{A}$ and $D_{B}$ obtained for a six-span beam made up by two sections of three elements of stiffness $\kappa_{1}^{*}$ (left) and $\kappa_{12 \text {,max }}^{*}$ (right), respectively. The remarkable reduction of the transmitted vibrations obtained with this configuration is shown in Fig. 6d. The noteworthy result is that, except for the natural frequencies of the ideal undamped system, the vibration reduction concerns all the frequencies of interest. Such result is evidenced by the narrow spikes around the natural frequencies. Next, in Figs. 7a c, the displacement dynamic magnification factor $D$ along the six-bays made up by three 
sections of two elements of stiffness $\kappa_{1}^{*}, \kappa_{12, \max }^{*}$ and $\kappa_{2}^{*}$ is shown for three adjacent frequency ranges $(3.00 \leqslant \beta \leqslant 4.35,4.35 \leqslant \beta \leqslant 6.30,6.30 \leqslant \beta \leqslant 8.0$, respectively). For such an undamped structure, although the peaks of the resonance amplifications are ideally infinite, the dynamic amplification
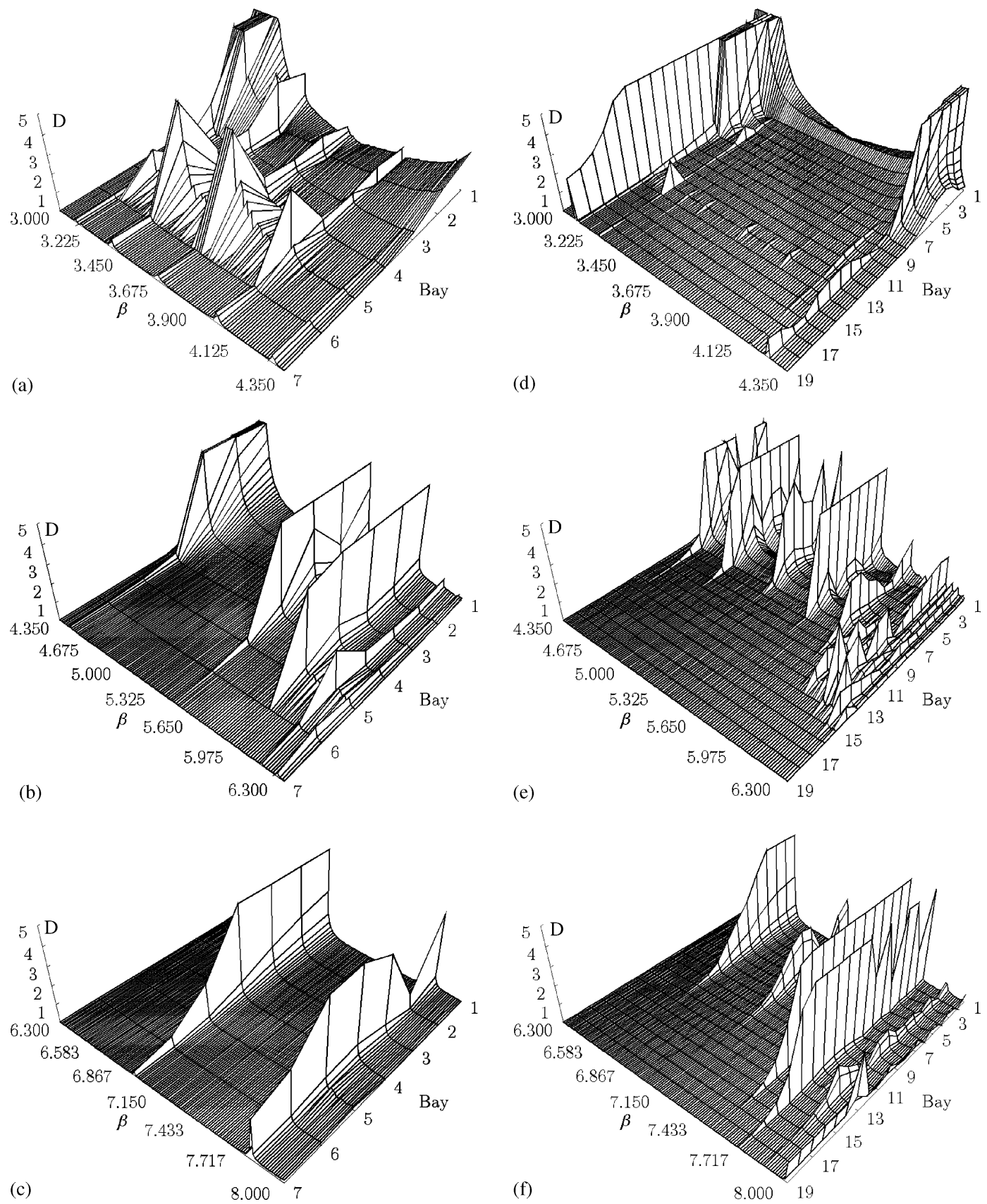

Fig. 7. Displacement dynamic magnification factors of optimal six span ((a) (c)) and 18 span ((d) (f)) piecewise periodic beams in the range $0<\beta<7.886$ : ((a), (d)) $3.0<\beta<4.35$; ((b), (e)) $4.35<\beta<6.30$; ((c), (f)) $6.30<\beta<8.0$. 
factor near resonance is lower and therefore resonance peaks narrow as one proceeds along the structure.

In order to highlight the influence of the total number of elements, the dynamic amplification factor for an 18-bays beam is considered (Figs. 7d f). Since the dimensionless parameter $\kappa$ must be kept constant, the dimensional length $l$ and stiffness $k_{t}$ must be decreased and increased, respectively, if compared with the six-bay beam. In one hand these figures show a faster vibration attenuation than the six-bay case; the attenuation occurring, as expected, for all frequencies but the natural ones. On the other hand, by increasing the number of elements the number of peaks increases as well. The plots refer to an excitation frequency sampling $\Delta \beta=0.01$, and the decaying trends of the resonance sharp peaks show that the resonances become narrower than the adopted sampling rate.

Further plots showing the effectiveness of the proposed design strategy are also reported in Figs. 8 and 9. The propagation bands of each optimal element considered are shown in Figs. 8a, c and $\mathrm{e}$ in terms of the propagation constant $\mu=\log \lambda$. In Figs. $8 \mathrm{~b}, \mathrm{~d}$ and $\mathrm{f}$, sections of the dynamic magnification factors at the value $D=1.5$ for 18-bay uniformly periodic beams composed by equal elements $\kappa_{1}^{*}, \kappa_{12, \max }^{*}$ and $\kappa_{2}^{*}$, respectively, are shown. It is rather evident that vibration transmission proceeds unaltered along the structure within the pass bands. On the other hand, as shown in Fig. 9a, by arranging in series the elements (with propagation characteristics depicted in Fig. 9b) only disturbances coinciding with the natural frequencies can propagate.

From the modal analysis standpoint the vibration transmission inhibition can be explained through the modal shape localization. However, only the first few modes have been found to be
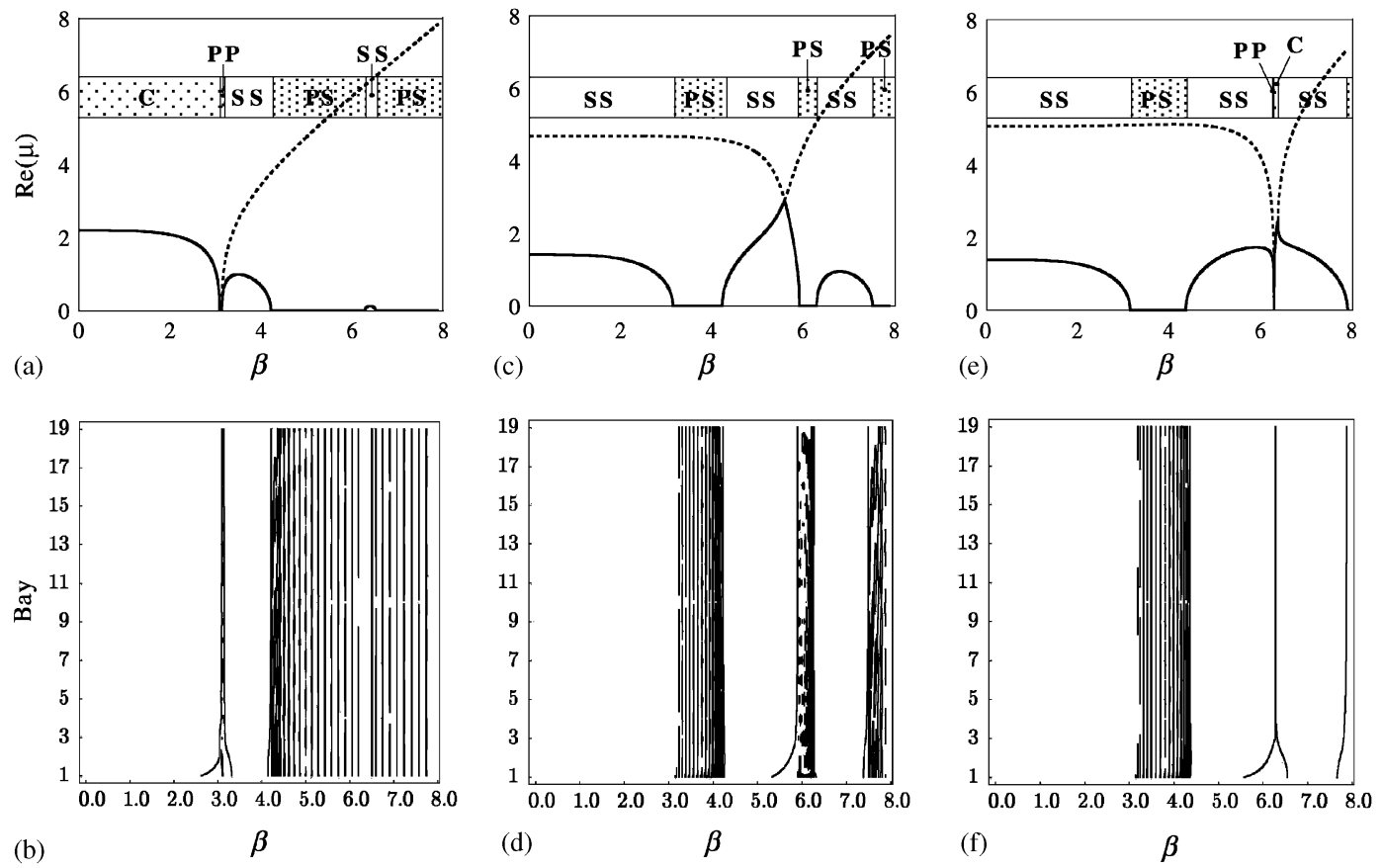

Fig. 8. Propagation bands of the first three optimal elements: (a) $\kappa_{1}^{*}$, (c) $\kappa_{12, \max }^{*}$, (e) $\kappa_{2}^{*}$. ((b), (d), (f)) Corresponding sections of the dynamic amplification factor at $D=1.5$ for 18 bay periodic beams. 

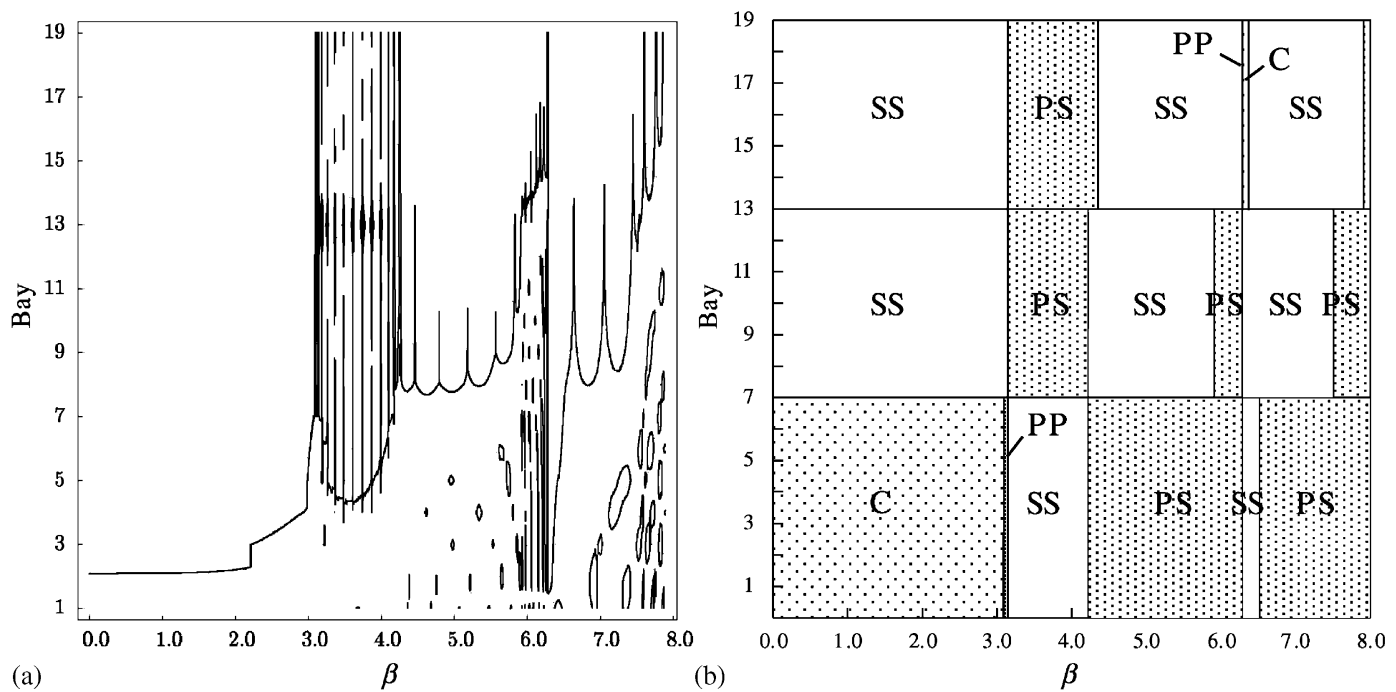

Fig. 9. Propagation along the optimal piecewise periodic beam in the range $0<\beta<7.886$ : (a) section of the displacement dynamic magnification at $D=0.1$, (b) propagation regions.

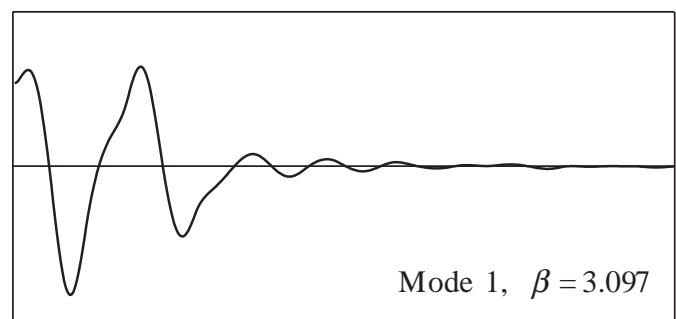

(a)

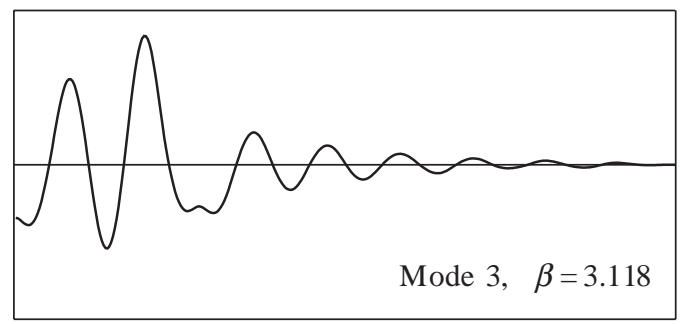

(c)

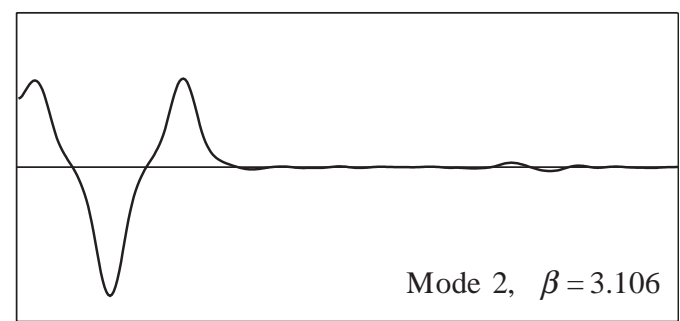

(b)

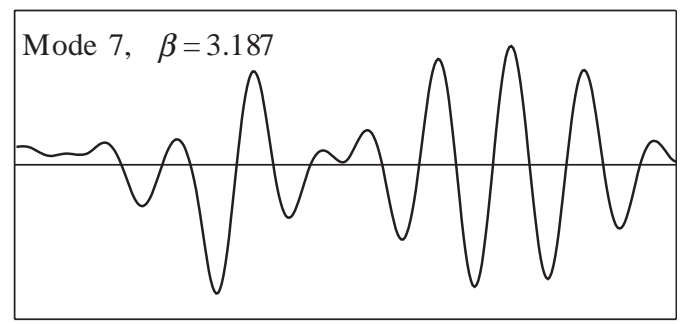

(d)

Fig. 10. ((a) (c)) Localized modal shapes; (d) non localized modal shape.

localized (see e.g. Fig. 10), therefore the high-frequency inhibition could solely be explained through the cumulative effect of different closely spaced modes; that is to say, modal analysis does not highlight the phenomenon. The above-mentioned localization pertaining to only the first few modes is in agreement with Ref. [11], since periodic structures with a finite number of elements are considered and piecewise periodicity acts as spatially dependent large imperfections in continuous structures. 


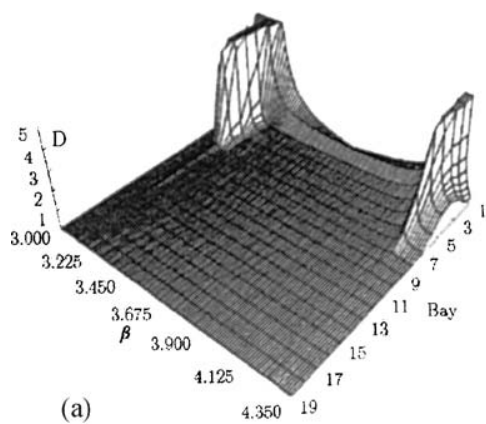

(a) $4.350 \quad 19$

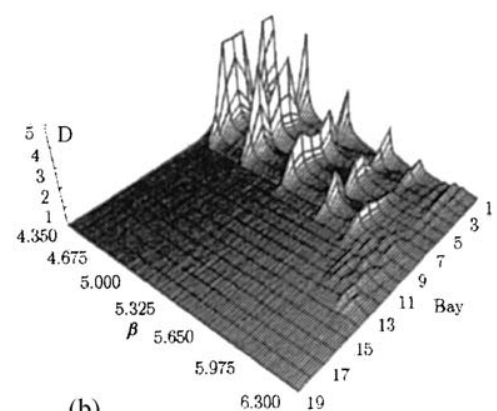

(b)

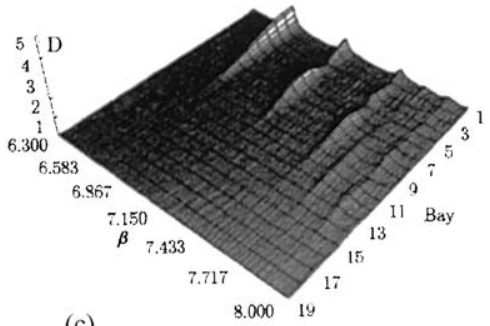

(c)

Fig. 11. Displacement dynamic magnification factors of the optimal 18 span piecewise periodic damped beam in the range $0<\beta<7.886$; damping factor $\eta=0.01$ : (a) $3.0<\beta<4.35$; (b) $4.35<\beta<6.30$; (c) $6.30<\beta<8.0$.

Table 2

Integral of the response at the final node of 18 bay periodic structures $(0 \leqslant \beta \leqslant 7.886)$; optimal sequence $\kappa_{1}^{*} \kappa_{12, \max }^{*} \kappa_{2}^{*}$

\begin{tabular}{|c|c|c|c|}
\hline Sections & Combinations & $\eta=1 \%$ & $\eta=2 \%$ \\
\hline 3 & 666 & $9.33 \mathrm{E}$ & $5.27 \mathrm{E}$ \\
\hline 3 & 477 & $1.68 \mathrm{E}$ & $1.03 \mathrm{E}$ \\
\hline 3 & 288 & $4.96 \mathrm{E}$ & $3.31 \mathrm{E}$ \\
\hline 3 & 747 & $6.59 \mathrm{E}$ & $3.87 \mathrm{E}$ \\
\hline 3 & 828 & $8.38 \mathrm{E}$ & $4.53 \mathrm{E}$ \\
\hline 3 & 774 & $1.17 \mathrm{E}$ & $6.95 \mathrm{E}$ \\
\hline 3 & 882 & $2.98 \mathrm{E}$ & $1.63 \mathrm{E}$ \\
\hline 1 & 1800 & 1.66 & 1.04 \\
\hline 1 & $\begin{array}{lll}0 & 18 & 0\end{array}$ & 1.59 & $6.94 \mathrm{E}$ \\
\hline 1 & $\begin{array}{lll}0 & 0 & 18\end{array}$ & $4.96 \mathrm{E}$ & $2.95 \mathrm{E}$ \\
\hline
\end{tabular}

Damped vibrations are then considered by introducing an hysteretic damping factor $\eta$ in the Euler beam equation of motion. Fig. 11 shows the dynamic amplification factor for the damped 18-bay optimal piecewise periodic beam with $\eta=0.01$. Besides the expected reduction of the resonance amplification, vibration transmission is now inhibited at all frequencies including the natural ones.

The influence of the number of elements in each optimal section along the structure is eventually investigated. A set of numerical tests has been carried out on a beam assuming different combinations of the number of elements in each section while keeping fixed the overall number of 18 bays. Under damped forced vibrations, the integral of the response at the final node, evaluated on the design frequency range $0<\beta<7.886$, is reported in Table 2 . In one hand, it can be inferred that once the optimal sequence is adopted, the number of elements in each section does not significantly affect the overall achievable vibration reduction since the differences in Table 2 concern small numbers. On the other hand, it can be noticed that the optimization of the number of elements in each section provides with vibration reductions larger or comparable with those 
obtained by a $1 \%$ damping increase in non-optimized sequences. If piecewise periodicity is removed by composing the periodic beam with only one optimal section, the amount of vibration reduction decreases by at least two order of magnitudes.

\section{Conclusions}

The design of bi-coupled periodic structures aiming at reducing the transmitted vibrations by optimally selecting piecewise periodicity has been proposed in this work. The design is conceived by simply combining the minimum number of dissimilar elements such that the union of the stop and complex propagation bands of each element covers the given excitation frequency bandwidth. The optimal combination of elements is derived from bi-dimensional analytical maps of the single unit free-wave propagation domains without the need of any calculation. The amount of vibration reduction increases with the number of elements in each section composing the optimal piecewise periodic structure; however, the more the elements the more the resonances arising in a given frequency range. The vibration analysis has been conducted relying on the wave vectors computational scheme whose main steps have also been illustrated. The effectiveness of the proposed design strategy has been confirmed through parametric numerical investigations.

\section{Appendix A. Scattering and reflection matrices}

Without loss of generality, the scattering matrix $\mathbf{S}$ is derived with reference to the interface $j$ of the scheme shown in Fig. 5. The transformation from state to wave vectors at node $j$ located between nodes $i$ and $k$ gives

$$
\left(\begin{array}{c}
\mathbf{x}_{j i} \\
\mathbf{f}_{j i}
\end{array}\right)=\mathbf{U}_{1}\left(\begin{array}{c}
\mathbf{r}_{j i} \\
\mathbf{l}_{j i}
\end{array}\right), \quad\left(\begin{array}{c}
\mathbf{x}_{j k} \\
\mathbf{f}_{j k}
\end{array}\right)=\mathbf{U}_{2}\left(\begin{array}{c}
\mathbf{r}_{j k} \\
\mathbf{l}_{j k}
\end{array}\right) .
$$

Continuity and equilibrium imply that $\left(\mathbf{x}_{j i}, \mathbf{f}_{j i}\right)=\left(\mathbf{x}_{j k}, \mathbf{f}_{j k}\right)$ so that

$$
\mathbf{U}_{1}\left(\begin{array}{c}
\mathbf{r}_{j i} \\
\mathbf{l}_{j i}
\end{array}\right)=\mathbf{U}_{2}\left(\begin{array}{c}
\mathbf{r}_{j k} \\
\mathbf{l}_{j k}
\end{array}\right) \text {. }
$$

In order to express the outgoing waves in terms of the incoming ones, the matrices $\mathbf{U}_{i}, i=1,2$ are partitioned

$$
\mathbf{U}_{i}=\left[\begin{array}{ll}
\mathbf{U}_{i, 11} & \mathbf{U}_{i, 12} \\
\mathbf{U}_{i, 21} & \mathbf{U}_{i, 22}
\end{array}\right], \quad i=1,2
$$

and the following matrices are assembled:

$$
\mathbf{S}_{A}=\left[\begin{array}{ll}
\mathbf{U}_{1,12} & \mathbf{U}_{2,11} \\
\mathbf{U}_{1,22} & \mathbf{U}_{2,21}
\end{array}\right], \quad \mathbf{S}_{B}=\left[\begin{array}{ll}
\mathbf{U}_{1,11} & \mathbf{U}_{2,12} \\
\mathbf{U}_{1,21} & \mathbf{U}_{2,22}
\end{array}\right]
$$


leading to the sought $(2 n \times 2 n)$ scattering matrix

$$
\mathbf{S}_{A}^{-1} \mathbf{S}_{B}=\mathbf{S}
$$

The reflection matrices $\mathbf{R}$ are also derived referring to the boundary condition of nodes $i$ and $k$ in Fig. 5. Such matrices are obtained by expressing the boundary conditions in terms of wave coordinates

$$
\begin{aligned}
& \mathbf{B}_{r, i} \mathbf{r}_{i j}+\mathbf{B}_{l, i} \mathbf{l}_{i j}=\mathbf{f}_{i}, \\
& \mathbf{B}_{r, k} \mathbf{r}_{k j}+\mathbf{B}_{l, k} \mathbf{l}_{k j}=\mathbf{0},
\end{aligned}
$$

where the entries in the matrices $\mathbf{B}$ are given by elements of the matrices $\mathbf{U}_{i}$ varying according to the type of constraint. Eq. (10) is obtained by rearranging Eq. (A.6); the reflections matrices $\mathbf{R}$ and the matrix $\mathbf{D}$ are given by

$$
\mathbf{R}_{i}=-\mathbf{B}_{l, i}^{-1} \mathbf{B}_{r, i}, \quad \mathbf{R}_{k}=-\mathbf{B}_{r, k}^{-1} \mathbf{B}_{l, k}, \quad \mathbf{D}=\mathbf{B}_{l, i}^{-1} .
$$

\section{References}

[1] R.S. Langley, N.S. Bardell, P.M. Loasby, The optimal design of near-periodic structures to minimize vibration transmission and stress levels, Journal of Sound and Vibration 207 (1997) 627-646.

[2] G.H. Koo, Y.S. Park, Vibration reduction by using periodic supports in a piping system, Journal of Sound and Vibration 210 (1998) 53-68.

[3] D. Richards, D.J. Pines, Passive reduction of gear mesh vibration using a periodic drive shaft, 42nd AIAA SDM Conference, Seattle, WA, 2001.

[4] A. Baz, Active control of periodic structures, Journal of Vibration and Acoustics 123 (2001) 472-479.

[5] F. Romeo, A. Luongo, Invariant representation of propagation properties for bi-coupled periodic structure, Journal of Sound and Vibration 257 (2002) 869-886.

[6] A.H. von Flotow, Disturbance propagation in structural networks, Journal of Sound and Vibration 106 (1986) 433-450.

[7] Y. Yong, Y.K. Lin, Propagation of decaying waves in periodic and piecewise periodic structures of finite length, Journal of Sound and Vibration 129 (1989) 99-118.

[8] G.Q. Cai, Y.K. Lin, Wave propagation and scattering in structural networks, Journal of Engineering Mechanics 117 (1991) 1555-1574.

[9] G. Sen Gupta, Natural flexural waves and the normal modes of periodically-supported beams and plates, Journal of Sound and Vibration 13 (1970) 89-101.

[10] M.G. Faulkner, D.P. Hong, Free vibrations of mono-coupled periodic system, Journal of Sound and Vibration 99 (1985) 29-42.

[11] A. Luongo, Mode localization in dynamics and buckling of linear imperfect continuous structures, Nonlinear Dynamics 25 (2001) 133-156. 